\title{
Amino-modified periodic mesoporous biphenylene-silica
}

\author{
Mirtha A.O. Lourenço a, Alvaro Mayoral ${ }^{\mathrm{c}}$, Isabel Díaz ${ }^{\mathrm{d}}$, Ana Rosa Silva ${ }^{\mathrm{b},{ }^{* *} \text {, }}$ \\ Paula Ferreira ${ }^{\text {a, * }}$ \\ a CICECO Aveiro Institute of Materials, Department of Materials \& Ceramic Engineering, University of Aveiro, 3810-193 Aveiro, Portugal \\ ${ }^{\mathrm{b}}$ CICECO Aveiro Institute of Materials, Department of Chemistry, University of Aveiro, 3810-193 Aveiro, Portugal \\ ${ }^{\mathrm{c}}$ Laboratorio de Microscopias Avanzadas, Instituto de Nanociencia de Aragon, Universidad de Zaragoza, 50018 Zaragoza, Spain \\ ${ }^{\mathrm{d}}$ Instituto de Catálisis y Petroleoquímica, CSIC, 28049 Madrid, Spain
}

\section{A R T I C L E I N F O}

\section{Article history:}

Received 10 February 2015

Received in revised form

14 June 2015

Accepted 19 June 2015

Available online 26 June 2015

\section{Keywords:}

Catalysis

PMO

Biphenylene

Knoevenagel condensation reaction

Amine

\begin{abstract}
A B S T R A C T
The amination reaction of biphenylene moieties in crystal like mesoporous silica (Bph PMO) is suc cessfully achieved with a density of $3.17 \mathrm{mmol} \mathrm{g}{ }^{1}$. The amination occurs approximately in every aro matic ring of the biphenylene bridge in the position 2 and $2^{\prime}$, leading to above $50 \%$ of the biphenylene group di aminated. The structural integrity of the synthetized material is preserved during the strong acid treatment to achieve the amine funcionalization of Bph PMO. The aminated mesoporous organo silica is highly active (initial turnover frequency $2193 \mathrm{~h} \mathrm{~h}^{1}$ or $37 \mathrm{~min}^{1}$ ) and almost $100 \%$ selective in the Knöevenagel condensation. Moreover, the amine modified periodic mesoporous biphenylene silica is effective for direct metal coordination.
\end{abstract}

(c) 2015 Elsevier Inc. All rights reserved.

\section{Introduction}

Periodic mesoporous organosilicas (PMOs) [1] have been calling much attention due to their promising applications, such as catal ysis, adsorption, molecular recognition, electronic and optical de vices. PMO materials are remarkable for their high specific surface areas and pore volumes, narrow distribution of pore sizes and enhanced thermal stability [2-5]. The alternating hydrophobic/ hydrophilic moieties along the mesochannel walls give to PMOs improved performance for example in catalytic reactions in which water may be formed and contribute to the deactivation of the catalytic site [6,7]. Furthermore, the organic bridge of the bisily lated precursor used to prepare the PMO can be changed promoting the creation of different features and interfacial interactions and tuning of the properties of the material for the desired application [8]. The ethane [2,9], ethylene [9,10], benzene [11], $p$ biphenylene [12] and thiophene [13] bridge were been successfully incorporated into the PMOs framework. Inagaki et al. [11] described the synthesis of phenylene PMO (Ph PMO), in 2002. This material had a high

\footnotetext{
* Corresponding author. Tel.: +351 234401419; fax: +351 234401470.

** Corresponding author. Tel.: +351 234401417; fax: +351 234401470.

E-mail addresses: ana.rosa.silva@ua.pt (A.R. Silva), pcferreira@ua.pt (P. Ferreira).
}

impact due to the concomitant presence of both hexagonal bidi mensional pore arrangement and molecular scale order along the wall. Moreover, the post synthesis sulfonation of the aromatic ring of $\mathrm{Ph} \mathrm{PMO}$ was demonstrated in the same report. In 2008, the same group reported the amination of $28 \%$ of phenylene moieties of PMO in a two step chemical transformation procedure [14]. This func tionalization reaction consists first on a strong acid treatment to introduce the nitro groups in the phenyl rings followed by their reduction to amines. Here, we describe for the first time the ami nation of the $p$ biphenylene PMO (Bph PMO) in a two step reac tion: (i) nitration of the aromatic rings of the biphenylene bridge, and (ii) reduction of the nitro groups to primary amines. Bph PMO displays an equimolar ratio of aromatic rings to silica atoms, of fering the opportunity to incorporate a large amount of functional groups on the material surface, which makes this material more interesting for catalysis or adsorption applications than the phenylene PMO. Moreover, Bracco et al. [15] reported an amphi dynamic behavior of the highly ordered hexagonal Bph PMO and demonstrated the existence of parallel and aligned biphenylene bridge along the mesochannel axes. The introduction of functional groups, which possess polarity may turn this materials important for application as molecular rotors. This material is tested as cata lyst in the Knoevenagel condensation and efficiency followed along consecutive cycles. 


\section{Experimental}

\subsection{Bph PMO synthesis and functionalization}

The Bph PMO was prepared following a well defined method (Scheme 1) [12]. 4,4' bis(triethoxysilyl)biphenylene (BTEBP, Aldrich, 95\%) precursor was hydrolyzed and condensed around a

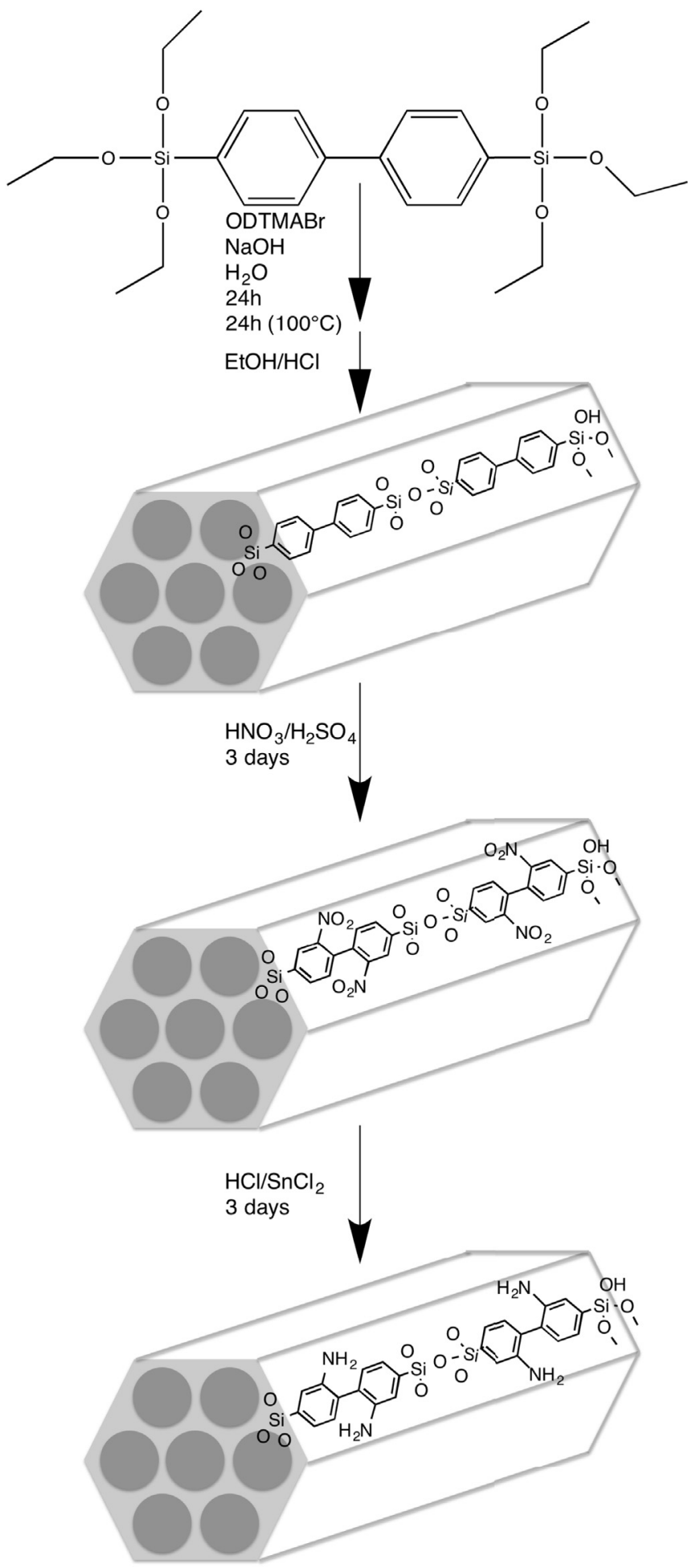

Scheme 1. Representation of the two steps functionalization reaction to achieve $\mathrm{NO}_{2}$ Bph-PMO and $\mathrm{NH}_{2}$-Bph-PMO materials. supramolecular structure agent, the octadecyltrimethylammonium bromide template (ODTMABr, Aldrich, $98 \%$ ). After $24 \mathrm{~h}$ of stirring at room temperature, followed by $24 \mathrm{~h}$ hydrothermal treatment at $100{ }^{\circ} \mathrm{C}$, the powder was recovered by filtration, washed with water and dried at $60{ }^{\circ} \mathrm{C}$. The template was extracted using an etha nol- $\mathrm{HCl}$ solution. The incorporation of functional amino groups into the phenylene rings (to obtain $\mathrm{NH}_{2}$ Bph PMO) was achieved upon two step treatment with very strong acid solutions of $\mathrm{HNO}_{3}-\mathrm{H}_{2} \mathrm{SO}_{4}$ and $\mathrm{SnCl}_{2}-\mathrm{HCl}$ using the same procedure of Ohashi et al. [14].

\subsection{Characterization of materials}

The physical and chemical properties of Bph PMO and func tionalized materials were evaluated by powder $\mathrm{X}$ ray diffraction (PXRD), low temperature $\left(196{ }^{\circ} \mathrm{C}\right)$ nitrogen adsorp tion-desorption isotherms, transmission electron microscopy (TEM), ${ }^{29} \mathrm{Si}$ MAS and CP MAS NMR, ${ }^{13} \mathrm{C}$ CP MAS NMR and FTIR spectroscopies. Description of the experimental conditions is pre sented at the Electronic Supplementary Information (ESI).

\subsection{Catalytic experiments}

All the catalytic reactions of the aminated material were per formed in batch reactors at atmospheric pressure and with con stant stirring. The Knoevenagel condensation was performed at $60{ }^{\circ} \mathrm{C}$ using $1.00 \mathrm{mmol}$ benzaldehyde, $1.00 \mathrm{mmol}$ malononitrile, $0.50 \mathrm{mmol}$ of chlorobenzene (internal standard) and 30 or $80 \mathrm{mg}$ of Bph PMO in $4.00 \mathrm{ml}$ of toluene, under inert atmosphere. The re action was followed by gas chromatography (GC, Bruker 450 GC) by periodically withdrawing aliquots from the reaction mixture. At the end of the reactions the material was isolated by filtration over $0.22 \mu \mathrm{m}$ nylon membranes and extensively washed with toluene, dried under vacuum at $60{ }^{\circ} \mathrm{C}$ and reused in another cycle using the same experimental procedure. A control experiment was also performed using the same experimental procedure, but without addition of the mesoporous organosilica. The reaction product was confirmed by GC-MS.

\section{Results and discussion}

\subsection{Structural and morphological characterization}

The Bph PMO was prepared and its aromatic rings were modi fied according to Scheme 1 to form $\mathrm{NO}_{2}$ Bph PMO and $\mathrm{NH}_{2}$ Bph PMO. PXRD, TEM and $\mathrm{N}_{2}$ sorption isotherms were used to evaluate the structural order and the textural physical properties of the pristine and functionalized Bph PMO materials.

Fig. 1 shows the PXRD patterns of Bph $\mathrm{PMO}, \mathrm{NO}_{2} \mathrm{Bph} \mathrm{PMO}$ and $\mathrm{NH}_{2}$ Bph PMO. All patterns are assigned a 2D hexagonal porous lattice with a space group $p 6 \mathrm{~mm}$. The first strong low angle peak is assigned to the (100) reflection is observed at a spacing $d \quad 4.96 \mathrm{~nm}$ for the parent Bph PMO and at $d \quad 4.70 \mathrm{~nm}$ for the $\mathrm{NH}_{2}$ Bph PMO (Table S1, ESI). The hexagonal unit cell lattice parameters, $a$, were calculated from the $d_{100}$ reflection using the equation $a \quad 2 d_{100} / \sqrt{ } 3$ and are equal to $5.73 \mathrm{~nm}$ for the Bph PMO and $5.42 \mathrm{~nm}$ for the $\mathrm{NH}_{2}$ Bph PMO showing that a slightly contraction of the unit cell occurs during the amination reaction.

The molecular scale periodicity for the Bph PMO is observed through the peaks at $d \quad 1.197,0.596,0.396,0.297$ and $0.242 \mathrm{~nm}$, highlighted by arrows. Similar peaks are observed for the $\mathrm{NO}_{2} \mathrm{Bph}$ PMO and $\mathrm{NH}_{2}$ Bph PMO. Both meso and molecular scale period icity are preserved after nitration and subsequent reduction of the nitro to the amine group. 

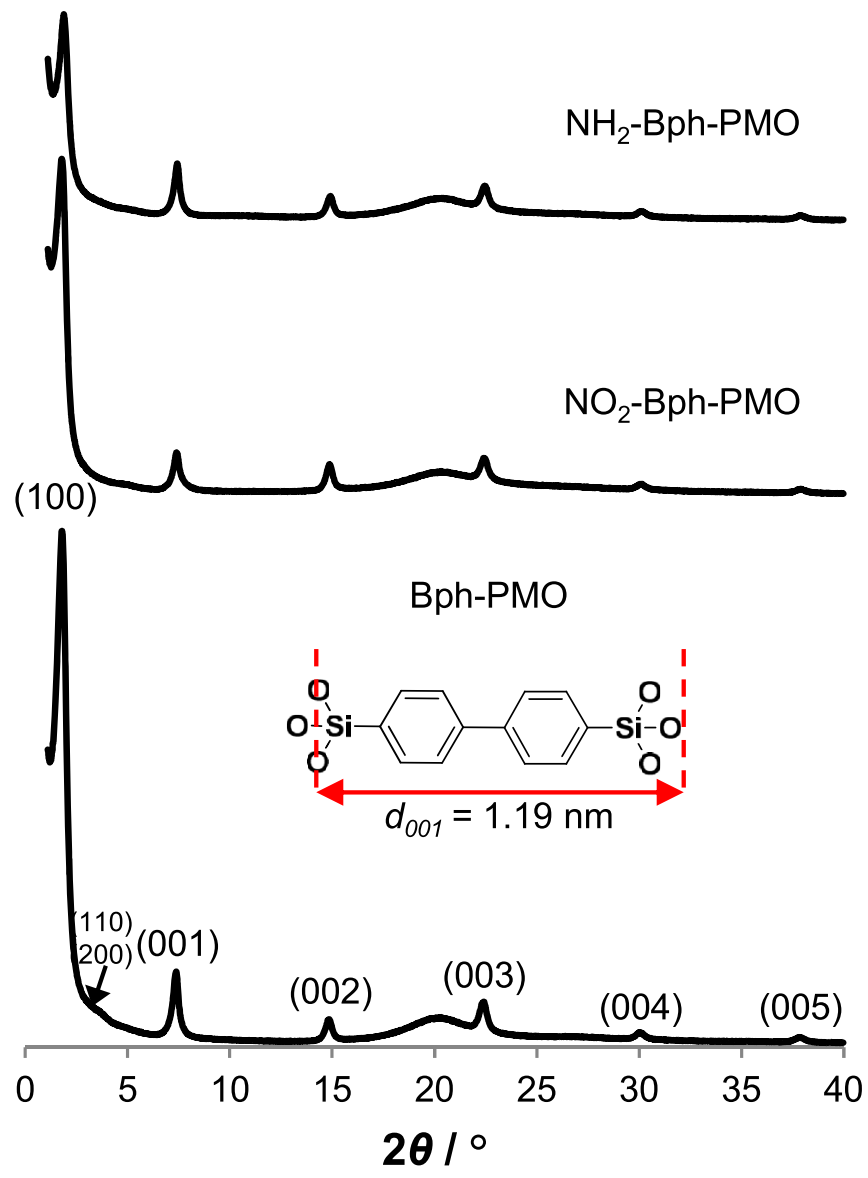

Fig. 1. X-ray diffraction patterns of Bph-PMO, $\mathrm{NO}_{2}$-Bph-PMO and $\mathrm{NH}_{2}$-Bph-PMO.
The electron microscopy images (Fig. 2) corroborate the local hexagonal mesoporous order of the channels in both parent and amine functionalized Bph PMO materials with pore diameters of 2.3 and $2.5 \mathrm{~nm}$, respectively. The molecular scale periodicity is also observed in both materials (Fig. 2c, e and f). The Bph PMO and $\mathrm{NH}_{2}$ Bph PMO display similar $d$ medium range spacing of $c a .1 .19 \mathrm{~nm}$, which are in agreement with the XRD patterns. It is also possible to note that the Bph PMO has a tendency to form particles with a helical form (Fig. 2a). This tendency remains after the amination of the biphenylene moieties (Fig. 2d).

The $\mathrm{N}_{2}$ sorption isotherms (Fig. S1, ESI) confirm the type IV isotherms (IUPAC classification) for Bph PMO typically observed for conventional mesoporous materials such as MCM 41. After func tionalization of the Bph PMO, the capacity of the materials was significantly reduced as can be observed in Fig. S1, (ESI). All syn thetized materials present a narrow distribution of pore sizes observed by the PSD curves on the inset of Fig. S1. The introduction of the amine group into the Bph PMO channels provided a reduc tion of the pore size from $3.27 \mathrm{~nm}$ for the parent Bph PMO to $3.02 \mathrm{~nm}$ for $\mathrm{NH}_{2}$ Bph PMO (Fig. S1 and Table S1, ESI).

The nitration and the amination of the biphenylene moieties of Bph PMO are supported by ${ }^{13} \mathrm{C}$ CP MAS NMR spectra (Fig. 3). The ${ }^{13} \mathrm{C}$ CP MAS NMR spectrum of Bph PMO presents four resonances at $c a .126,130,135$ and $141 \mathrm{ppm}$ assigned to the $\mathrm{sp}^{2}$ carbons from the biphenylene group. The nitration of the biphenylene moieties outcomes in five resonances at ca. 126, 130, 135, 140 and 147 ppm indicating the presence of two nitro groups linked to the biphe nylene moieties in symmetric positions. This is enlightened by the existence of two benzene rings linked to each other in the Bph PMO, both available to be nitrated [16]. The phenyl group is an electron donating group, being more stable in the resonance forms of ortho and para positions in the biphenylene group. In this way, the nitration of the biphenylene moieties occurs mainly at ortho and para positions rather than at meta position, which is
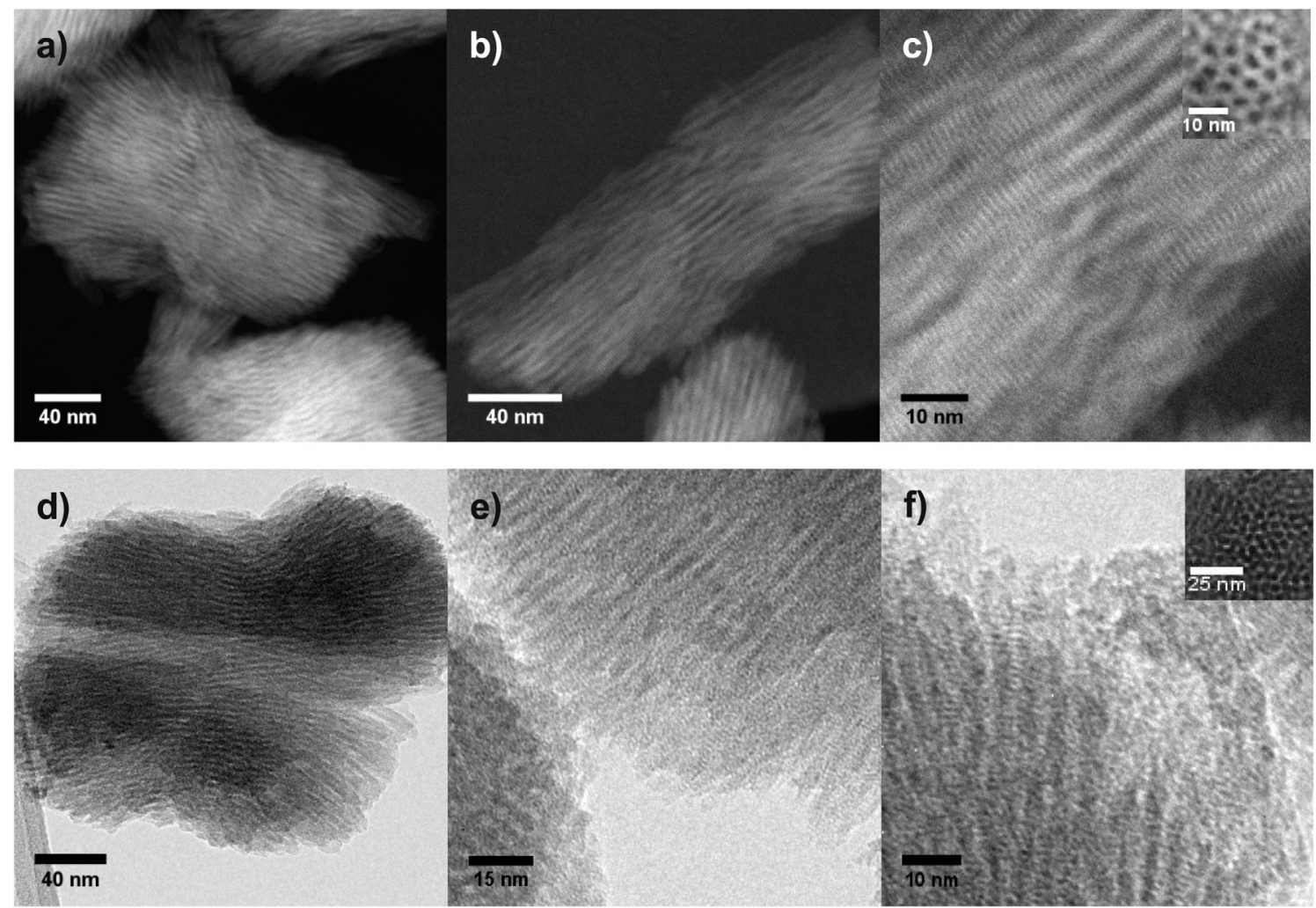

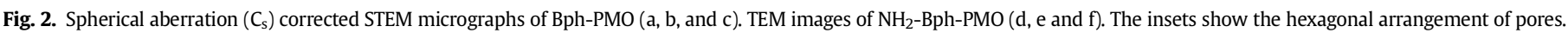




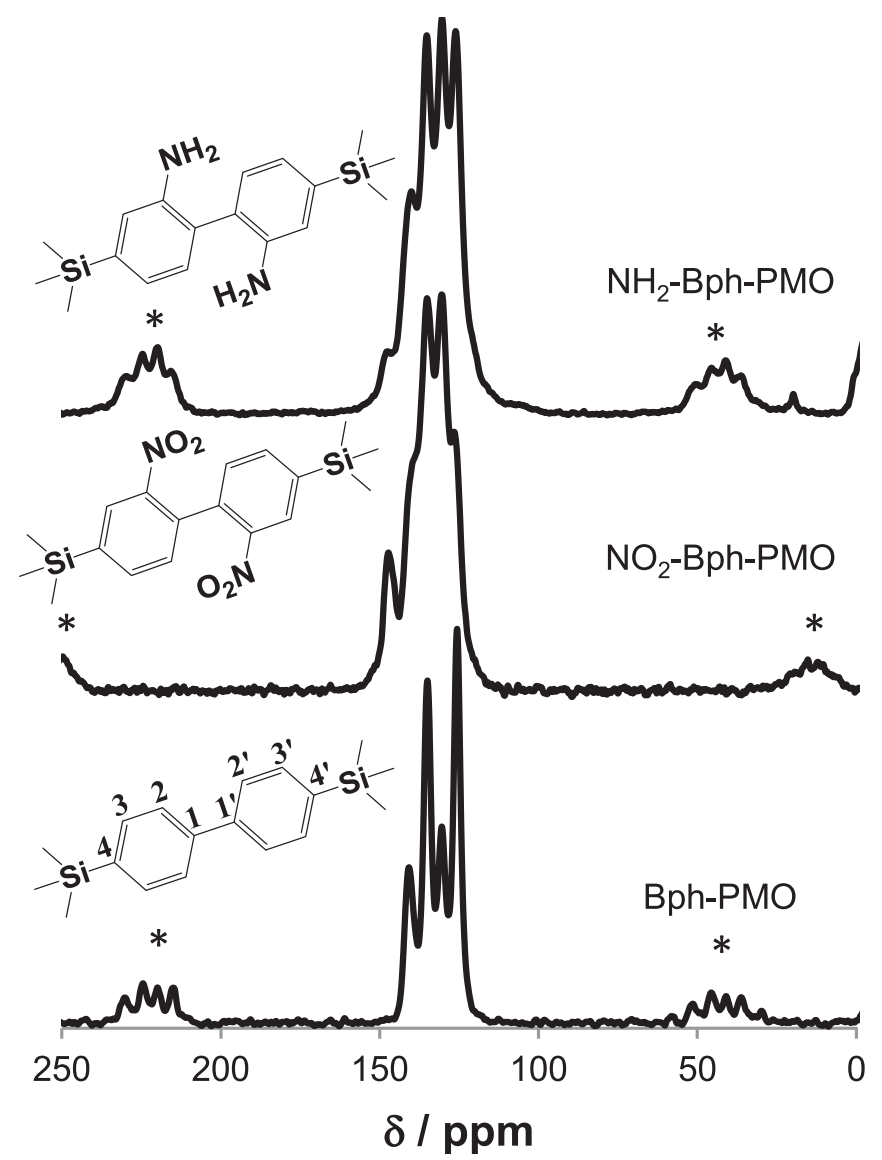

Fig. 3. ${ }^{13} \mathrm{C} C P-M A S$ NMR spectra of Bph-PMO, $\mathrm{NO}_{2}$-Bph-PMO and $\mathrm{NH}_{2}$-Bph-PMO.

explained by the resonance structures of the intermediates. The 4 and $4^{\prime}$ position of the biphenylene moieties are occupied by the silica group, that correspond to the para direction, the electro philic attack of the biphenylene moieties to the nitric acid hap pens essentially in the position 2 (ortho position). The reactivity of the biphenylene group with a nitro group linked in the ortho position of the phenyl substituent is affected. The nitro phenyl group is also ortho para directing as the phenyl group but it is also deactivating, rendering the electrophilic attack of the 2 nitrodiphenyl to the nitric acid slower [17]. Moreover, the nitra tion reaction is made using $\mathrm{HNO}_{3}$ and $\mathrm{H}_{2} \mathrm{SO}_{4}$ during 3 day at room temperature, which increases the possibility to incorporate two nitro groups in the phenylene moieties of the Bph PMO. Having into account the resonance forms of the 2 nitrodiphenyl PMO, the electrophilic attack to the nitric acid arises in the $2^{\prime}$ position affording the di nitrated Bph PMO. This is supported by the ${ }^{13} \mathrm{C} C \mathrm{P}$ MAS NMR.

The reduction of the nitro groups to the amine groups leads to some changes in the ${ }^{13} \mathrm{C}$ NMR spectra. Although, the chemical shift of the five resonances peaks remains.

The ${ }^{15} \mathrm{~N}$ CP MAS NMR spectrum (Fig. S2, ESI) of $\mathrm{NH}_{2}$ Bph PMO displays a single peak at $c a$. $325 \mathrm{ppm}$, which was assigned to a primary amine group $\left(\mathrm{NH}_{2}\right)$ linked to an aromatic ring.

Fig. S3 (ESI) shows the ${ }^{29} \mathrm{Si}$ MAS and CP MAS NMR spectra of all materials. The pristine Bph PMO exhibits peaks at ca. 81, 71 and $61 \mathrm{ppm}$ assigned to, respectively, $\mathrm{T}^{3}, \mathrm{~T}^{2}$ and $\mathrm{T}^{1}$ organosiliceous species $\left[\mathrm{T}^{\mathrm{m}} \quad \mathrm{RSi}(\mathrm{OSi})_{\mathrm{m}}(\mathrm{OH})_{3-\mathrm{m}}\right]$. After amine functionalization of the Bph PMOs, no $\mathrm{Q}^{\mathrm{n}}$ species $\left[\mathrm{Q}^{\mathrm{n}} \quad \mathrm{Si}(\mathrm{OSi})_{\mathrm{n}}(\mathrm{OH})_{4-\mathrm{n}}\right]$ are observed in the ${ }^{29} \mathrm{Si}$ NMR spectra, which shows that no carbon-silicon bond cleavage occurs during the amination reaction.

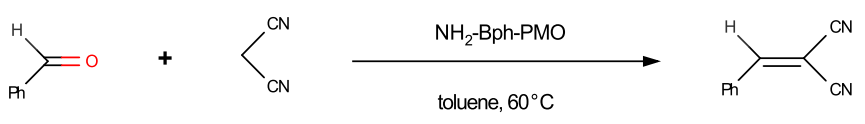

Fig. 4. Knoevenagel condensation of benzaldehyde with malononitrile.

The functionalization reaction of Bph PMOs was also followed by FTIR (Fig. S4, ESI). The first nitration was confirmed by the presence of $\mathrm{C}-\mathrm{N}$ stretching band at $1266 \mathrm{~cm}^{-1}$ and by two strong bands at 1352 and $1534 \mathrm{~cm}^{-1}$ that correspond to the $\mathrm{N}-\mathrm{O}$ stretching bands of the $\mathrm{NO}_{2}$ group. The reduction of the $\mathrm{NO}_{2}$ to the $\mathrm{NH}_{2}$ group was observed by the disappearance of the $\mathrm{N}-\mathrm{O}$ stretching bands and the presence of the $\mathrm{N}-\mathrm{H}$ bending band at $1608 \mathrm{~cm}^{-1}$ and the $\mathrm{N}-\mathrm{H}$ stretching modes of aromatic primary amines at 3379 and $3340 \mathrm{~cm}^{-1}$.

Elemental analyses of $\mathrm{NH}_{2}$ Bph PMO provide a nitrogen density close to $3.17 \mathrm{mmol} \mathrm{g}^{-1}$ ( $4.4 \%$ of $\mathrm{N}$ ), indicating that almost $46 \%$ of biphenylene bridges were di functionalized with the amine group or at least all biphenylene bridge has one amine group. The first hypothesis seems to be the one supported by the ${ }^{13} \mathrm{C} C P$ MAS NMR spectrum.

Fig. S5 (ESI) shows the TGA curves exhibits for all synthetized materials. The first weight loss corresponds to desorption of physisorbed water and occurs below $100{ }^{\circ} \mathrm{C}$. The thermal stability of the pristine Bph PMO material is up to $550{ }^{\circ} \mathrm{C}$. Above this tem perature the biphenylene moieties from the framework of the Bph PMO suffers decomposition and are released. After nitration or amination functionalization of the Bph PMO the thermal stability of the material decreases to $375{ }^{\circ} \mathrm{C}$ and $425^{\circ} \mathrm{C}$ for $\mathrm{NO}_{2}$ Bph PMO and $\mathrm{NH}_{2}$ Bph PMO, respectively.

\subsection{Catalytic tests}

Amine functionalized mesoporous materials have been studied as heterogeneous catalysts for the Knoevenagel condensation (Fig. 4) [14,18]. This is an important C-C coupling reaction of a carbonyl group with a compound containing an activated methy lene group (Fig. 4) and is widely used for the synthesis of fine chemicals and pharmaceuticles [18]. Thus the new aminated $\mathrm{NH}_{2}$ Bph PMO material was tested in the condensation of benzalde hyde and malonitrile at $60{ }^{\circ} \mathrm{C}$ in toluene (Fig. 3). The result is depicted in Fig. 5 and shows that this material is able to catalyze the Knoevenagel condensation using just $30 \mathrm{mg}$ of material

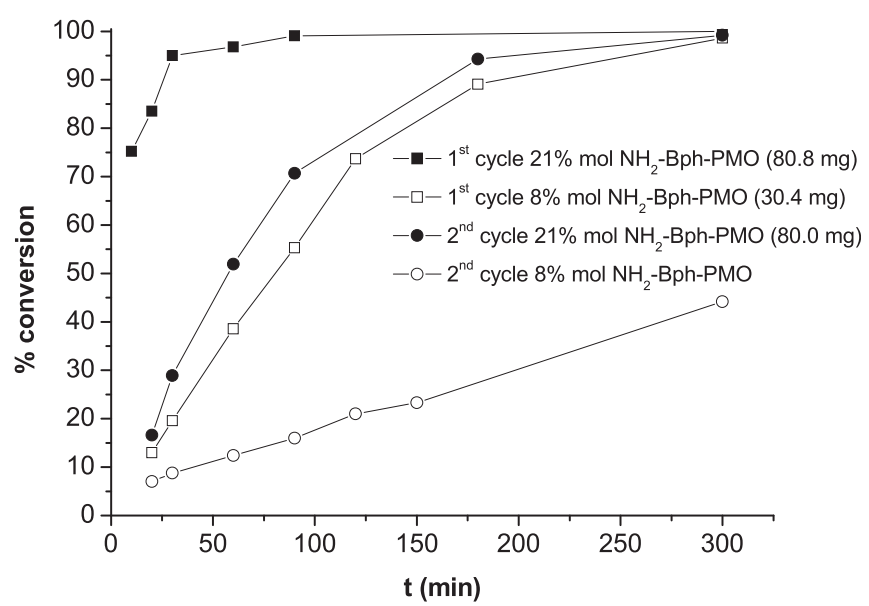

Fig. 5. Kinetic profiles for the Knoevenagel condensation of benzaldehyde with malononitrile using two different weights of $\mathrm{NH}_{2}$-Bph-PMO as heterogeneous catalyst. 
containing $0.07 \mathrm{mmol}$ of amino groups (7\% mol relative to the substrates). Using $80 \mathrm{mg}$ of material containing $0.18 \mathrm{mmol}$ of amino groups ( $18 \% \mathrm{~mol}$ relative to the substrates), the reaction proceeds much faster with high catalytic activity (initial turnover frequency $2193 \mathrm{~h}^{-1}$ or $37 \mathrm{~min}^{-1}$ ) and after $30 \mathrm{~min} 95 \%$ of the benzaldehyde is converted into the condensation product (Fig. 5). After 90 min almost full conversion is observed (with a turnover number of 481). It is noteworthy that the control experiment performed without the addition of the material also takes place but at residual rate and after 120 min of reaction only $6 \%$ of con version is observed. A minor quantity of carboxylic acid was only detected by GC-MS (not by GC) indicating that the reaction is almost 100\% selective towards the Knoevenagel condensation product (Fig. 4).

The catalyst was separated from the reaction media by simple filtration and reused in one more cycle. Reduction of the reaction rate is observed for both reactions catalyzed using 30 or $80 \mathrm{mg}$ of $\mathrm{NH}_{2}$ Bph PMO (Fig. 5). Although using $80 \mathrm{mg}$ of $\mathrm{NH}_{2}$ Bph PMO full substrate conversion is observed after $5 \mathrm{~h}$ of reaction, after $90 \mathrm{~min}$ only $59 \%$ of benzaldehyde is converted. Nevertheless, we also realized that deactivation is highly dependent on the amine den sity (Fig. S8) and that using $\mathrm{NH}_{2}$ Bph $\mathrm{PMO}$ with higher amine contents the deactivation was not so pronounced. The mechanism of the reaction has been described to proceed via imine formation at the surface of the materials, by Schiff condensation between the surface amino groups and benzaldehyde [18]. Then the acidic carbon of the methylene compound attacks the imine and the Knoevenagel condensation product is formed (Fig. 4) [18]. Since the reactions were performed using equimolar amounts of benz aldehyde and malononitrile the reaction rate slows down in the second use of the material due to slower attack of the methylene compound to the imines formed at the surface of the $\mathrm{NH}_{2} \mathrm{Bph}$ PMO material. Characterization of the material after two cycles of catalysis shows that there is a decrease in its surface area (from 605 to $397 \mathrm{~m}^{2} \mathrm{~g}^{-1}$, Fig. S1 and Table S1). The XRD is similar to the original material, showing that the mesostructure is not lost (Fig. S6). The FTIR of the used $\mathrm{NH}_{2}$ Bph PMO show two sharp bands at 1536 and $1607 \mathrm{~cm}^{-1}$ which are characteristic of imine groups (Fig. S7). The TG of the materials after catalysis also shows a $100{ }^{\circ} \mathrm{C}$ decrease in the thermal stability (Fig. S5), supporting the presence of the imines at the surface of the material. Thus the decrease in the surface area of the material (Table S1, ESI) may be due to pore blockage from the imines formed at the surface of the $\mathrm{NH}_{2}$ Bph PMO material. This may be enhanced by the kind of elliptical structure observed by TEM for the $\mathrm{NH}_{2}$ Bph PMO and has as ultimate consequence in terms kinetics the slow down of the reaction rate due to difficult substrate and product diffusion through the pore channels.

Deactivation of amine modified porous materials in the Knoevenagel condensation has already been observed in the literature on the tandem Knoevenagel condensation/allylic $\mathrm{N}$ alkylation by an amine modified MOF [19]. And deactivation in a second cycle was also observed, which was attributed to degra dation of the MOF [19]. Therefore the PMO might be more stable than MOF under the catalytic experimental conditions. MOF are usually microporous materials, whereas PMO are normally meso porous materials. Thus another advantage of the PMO materials when compared to MOF is that liquid phase reactions might pro ceed faster with lower diffusion limitations due to the bigger pore size. Nevertheless, comparisons between materials are not always straightforward as the quantity of active phase (amine) in the material is a very important factor (Fig. S8) and the turnover numbers, turnover frequencies or recycling data are not always provided for the same substrates or under the same experimental conditions.

\subsection{Metal coordination}

Amine modified materials can also be used for metal coordi nation. In order to do the proof of concept, we refluxed for $48 \mathrm{~h}$ a methanolic solution of a $0.0212 \mathrm{~g}$ of copper(II) salt (copper(II) tri flate) with $0.0510 \mathrm{~g}$ of the amine modified periodic mesoporous biphenylene silica. The material was filtered, thoroughly washed with several portions of methanol and then refluxed with fresh methanol for $6 \mathrm{~h}$ to ensure the removal of physisorbed $\mathrm{Cu}(\mathrm{II})$. After filtering and drying the material, with a greenish color, ICP analysis revealed that it contained $0.92 \%$ of $\mathrm{Cu}$. Thus the amine modified periodic mesoporous biphenylene silica can be used for direct metal coordination.

\section{Conclusions}

We demonstrated that well ordered amino functionalized pe riodic mesoporous biphenylenesilica was prepared with $4.4 \%$ of $\mathrm{N}$. This material showed high catalytic activity (initial turnover fre quency $2193 \mathrm{~h}^{-1}$ or $37 \mathrm{~min}^{-1}$ ) and almost $100 \%$ selectivity for the Knoevenagel condensation, typical of other aminated porous sup ports. Further reuse of the material in one more catalytic cycle leads to loss of catalytic activity. Other applications of this new material such as metal coordination or modification of the amino group towards molecular rotor applications are avenues of research to be explored.

\section{Acknowledgment}

This work was developed in the scope of the project CICECO Aveiro Institute of Materials (Ref. FCT UID/CTM/50011/2013), financed by National funds through the FCT/MEC and when applicable co financed by FEDER under the PT2020 Partnership Agreement. Authors are grateful to the Fundaçao para a Ciência e a Tecnologia (FCT), Fundo Europeu de Desenvolvimento Regional (FEDER), QREN COMPETE and the European Union, for funding the project FCOMP 010124 FEDER 015644 (PTDC/QUI-QUI/113678/ 2009). ARS and PF acknowledge FCT for IF/01300/2012 and IF/ 00327/2013, respectively. MAOL thanks the PhD grant SFRH/BD/ $80883 / 2011$. The research leading to these results has received funding from the European Union Seventh Framework Program under Grant Agreement 312483 - ESTEEM2 (Integrated Infra structure Initiative-I3).

\section{Appendix A. Supplementary data}

Supplementary data related to this article can be found at http:/ dx.doi.org/10.1016/j.micromeso.2015.06.026.

\section{References}

[1] S. Inagaki, S. Guan, Y. Fukushima, T. Ohsuna, J. Am. Chem. Soc. 121 (1999) 96119614.

[2] P. Van der Voort, D. Esquivel, E. De Canck, F. Goethals, I. Van Driessche, F.J. Romero-Salguero, Chem. Soc. Rev. 42 (2013) 39133955.

[3] F. Hoffmann, M. Froba, Chem. Soc. Rev, 40 (2011) 608620.

[4] W. Wang, J.E. Lofgreen, G.A. Ozin, Small 6 (2010) 26342642.

[5] N. Mizoshita, T. Tani, S. Inagaki, Chem. Soc. Rev. 40 (2011) 789800.

[6] A. Karam, J.C. Alonso, T.I. Gerganova, P. Ferreira, N. Bion, J. Barrault, F. Jérôme Chem. Commun. (2009) 70007002.

[7] R. Siegel, E. Domingues, R. De Sousa, F. Jérôme, C.M. Morais, N. Bion, P. Ferreira, L. Mafra, J. Mater. Chem. 22 (2012) 74127419.

[8] M. MacLachlan, T. Asefa, G. Ozin, Chemistry 6 (2000) 25072511.

[9] B. Melde, B. Holland, Chem. Mater. (1999) 33023308.

[10] T. Asefa, M.J. MacLachlan, N. Coombs, G.A. Ozin, Nature 402 (1999) 867871.

[11] S. Inagaki, S. Guan, T. Ohsuna, O. Terasaki, Nature 416 (2002) 304307.

[12] M.P. Kapoor, Q. Yang, S. Inagaki, J. Am. Chem. Soc. 124 (2002) 1517615177.

[13] C. Yoshina-Ishii, T. Asefa, N. Coombs, M.J. MacLachlan, G.A. Ozin, Chem. Commun. (1999) 25392540. 
[14] M. Ohashi, M.P. Kapoor, S. Inagaki, Chem. Commun. (2008) 841843.

[15] S. Bracco, A. Comotti, P. Valsesia, B.F. Chmelka, P. Sozzani, Chem. Commun. (Camb) (2008) 47984800.

[16] Y.F. Tai, C. Ji, C.J. Shi, W. Wang, X.H. Peng, 35 (2014) 12411243.
[17] J.G. Hoggett, R.B. Moodie, J.R. Penton, K. Schofield, Nitration and Aromatic Reactivity, first ed., Syndics of the Cambridge University Press, Cambridge, 1971.

[18] Y. Yang, H.F. Yao, F.G. Xi, E.Q. Gao, J. Mol. Catal. A Chem. 390 (2014) 198205

[19] P.V. Dau, S.M. Cohen, Inorg. Chem. 54 (2015) 31343138. 